\title{
Pyrogenic Carbon Lacks Long-Term Persistence in Temperate Arable Soils
}

\author{
Suzanne Lutfalla ${ }^{1,2}$, Samuel Abiven ${ }^{3}$, Pierre Barré ${ }^{2 *}$, Daniel B. Wiedemeier ${ }^{3}$, \\ Bent T. Christensen ${ }^{4}$, Sabine Houot ${ }^{1}$, Thomas Kätterer ${ }^{5}$, Andy J. Macdonald ${ }^{6}$, \\ Folkert van Oort ${ }^{1}$ and Claire Chenu ${ }^{1 *}$ \\ ${ }^{1}$ AgroParisTech, Institut National de la Recherche Agronomique, UMR 1402 ECOSYS, Thiverval Grignon, France, \\ ${ }^{2}$ Laboratoire de Géologie de l'ENS-PSL Research University-Centre National de la Recherche Scientifique UMR8538, Paris, \\ France, ${ }^{3}$ Department of Geography, University of Zurich, Zurich, Switzerland, ${ }^{4}$ Department of Agroecology, Aarhus \\ University, Aarhus, Denmark, ${ }^{5}$ Department of Ecology, Swedish University of Agricultural Sciences, Uppsala, Sweden, \\ ${ }^{6}$ Department of Sustainable Soils and Grassland Systems, Rothamsted Research, Harpenden, United Kingdom
}

\section{OPEN ACCESS}

Edited by:

Moritz Felix Lehmann, University of Basel, Switzerland

Reviewed by:

Carlos A. Sierra, Max Planck Institute for Biogeochemistry (MPG), Germany Caitlin E. Hicks Pries, Dartmouth College, United States

*Correspondence: Pierre Barré barre@geologie.ens.fr Claire Chenu claire.chenu@agroparistech.fr

\footnotetext{
Specialty section:

This article was submitted to Biogeoscience a section of the journal Frontiers in Earth Science
}

Received: 31 July 2017 Accepted: 09 November 2017 Published: 23 November 2017

Citation:

Lutfalla S, Abiven S, Barré $P$, Wiedemeier DB, Christensen BT, Houot S, Kätterer T, Macdonald AJ, van Oort F and Chenu C (2017) Pyrogenic Carbon Lacks Long-Term Persistence in Temperate Arable Soils. Front. Earth Sci. 5:96 doi: 10.3389/feart.2017.00096
Pyrogenic organic carbon (PyOC) derived from incomplete burning of biomass is considered the most persistent fraction of soil organic carbon (SOC), being expected to remain in soil for centuries. However, PyOC persistence has seldom been evaluated under field conditions. Based on a unique set of soils from five European long-term bare fallows (LTBF), i.e., vegetation-free field experiments, we provide the first direct comparison between PyOC and SOC persistence in temperate arable soils. We found that soil PyOC contents decreased more rapidly than expected from current concepts, the mean residence time (MRT) of native PyOC being just 1.6 times longer than that of SOC. At the oldest experimental site, $55 \%$ of the initial PyOC remained after 80 years of bare fallow. Our results suggest that while the potential for long-term $\mathrm{C}$ storage exists, the persistence of PyOC in soil may currently be overestimated.

Keywords: soil organic matter persistence, soil carbon sequestration, carbon cycle, pyrogenic carbon, climate change mitigation, long term bare fallows

\section{INTRODUCTION}

Pyrogenic Organic Carbon (PyOC) is a charred residue derived from the incomplete burning of biomass and is a native component of most soils. PyOC represents on average about $14 \%$ of the soil organic carbon (SOC) and ranges between 0 and $60 \%$ depending on the conditions of fire, soils and climate (Reisser et al., 2016). This corresponds to a stock of about $200 \mathrm{Pg}$ of C (Reisser et al., 2016), within the same order of magnitude as the global vegetation C stocks. Since PyOC inputs are mostly coming from fires, their occurrence and amount should be low as compared to uncharred organic material (i.e., litter). One reason for these high stocks can be related to the specific persistence of PyOC in terrestrial ecosystems.

Recent reviews suggest that the persistence of PyOC in soil may range from a few years to millennia (Singh et al., 2012a; Lehmann et al., 2015). These differences can be explained by the heterogeneity of PyOC material, but also by the different methods used to assess its presence in soils. While being a limited tool to assess the persistence of SOC (Sierra et al., 2017), mean residence time (MRT) is widely used to compare the different turnovers of organic materials. Assessing the MRT of PyOC is complex, because of the large time span that should be considered. Lehmann et al. (2015) described the existing approaches and their current limitations, among which: laboratory soil incubations, field setups, and space for time approaches. 
Laboratory soil incubations can be well constrained, however they are limited by their duration and their lack of litter inputs, plants, macro-fauna, micro-organisms variations and water, and temperature dynamics. They address mainly the first phase of PyOC mineralization and are mainly useful to compare PyOC to an uncharred material (Zimmerman, 2010; Singh et al., 2012b).

Field studies are more realistic but also suffer from different hurdles. Purposeful additions of PyOC in field setups share the advantages of controlled conditions with incubations and allow $\mathrm{C}$ budget, particularly in the case of isotopically labeled material, but only address few years of mineralization at maximum (Whitman et al., 2014). Calculating the PyOC balance of natural char dynamics is a way to address longer time scales (Lehmann et al., 2008; Lehndorff et al., 2014). It requires a well-known input of chars over time, estimated for a period that well exceeds MRT, and PyOC exports from other processes than mineralization must be small (Lehmann et al., 2015). These conditions are difficult to fulfill, and the MRT thus estimated is very dependent on the assumptions considered for PyOC inputs.

PyOC MRT can also be estimated via a space for time approach, also named chronosequence (Lehmann et al., 2015). It consists in sampling soils where PyOC entered the soil at different times in the past (Nguyen et al., 2009; Vasilyeva et al., 2011; Alexis et al., 2012), as a substitute for archived samples of the same site at different times. The advantage of this approach is that the MRT can be calculated over long periods, provided one can find samples from comparable environmental conditions, receiving a similar amount of PyOC at several year or decade intervals. These conditions are very difficult to find, and the pre-required hypothesis nearly impossible to validate.

As described above, field studies are more realistic than laboratory incubations to estimate the PyOC MRT, but the requirements regarding the inputs and the site are difficult to match. Long-term bare fallow (LTBF) is another field approach that has been successfully applied to different fractions of SOC (Barré et al., 2010; Paradelo et al., 2013). It consists in following over time (with archived samples) the degradation of organic material while the soil does not receive any new organic inputs (Rühlmann, 1999). This approach solves part of the shortcomings described above: there is no input considered in this setup, so no hypothesis about their amount and characteristics. Also, there is no need to speculate about the difference between sites as in the space for time approach since all samples originate from the same location. Compared to laboratory incubations, LTBF which have no litter inputs by design have the advantage of representing in situ environmental, climatic and biological conditions and preventing soils from being physically disturbed.

Our study is based on archived soils sampled in five European LTBF experiments that allowed us to study the corresponding decay rates of PyOC and SOC over several decades and under different temperate pedo-climatic settings (Barré et al., 2010; Table 1). In these experiments, soils do not receive any organic inputs and are kept free of vegetation by weeding (manual weeding and/or chemical weeding on a yearly basis) for up to 80 years. As the largest $\mathrm{C}$ inputs to soils are derived from plants, the total $\mathrm{C}$ inputs during the bare fallow period are negligible, thanks to the absence of plants. The vegetation-free setup of the LTBF experiments represents a unique opportunity for studying in situ the long-term turnover of PyOC and SOC in agricultural soils. Indeed, they provide information on initial stocks and subsequent decadal-scale changes without interference from fresh C inputs.

\section{MATERIALS AND METHODS \\ European Network of Long Term Bare Fallows (LTBF)}

Soil samples were taken from the archives of a European network of LTBF experiments located in five different sites (see Table 1). The experiments were originally initiated for agronomic studies on arable soil fertility. The characteristics of the five sites involved in this study have been described in Barré et al. (2010). For each LTBF experiment, we selected two adjacent field plot replicates (four replicates at Versailles). For analyses, we used archived soils sampled at the start of the bare fallow treatment and after different fallow periods (samples are air-dried and kept in the dark). Except at Rothamsted, there are no significant changes

TABLE 1 | Selected characteristics of the five LTBF sites.

\begin{tabular}{|c|c|c|c|c|c|}
\hline & Askov (DK) & Grignon (FR) & Rothamsted (UK) & Ultuna (SW) & Versailles (FR) \\
\hline Coordinates & $55^{\circ} 28 \mathrm{~N} 9^{\circ} 07 \mathrm{E}$ & $48^{\circ} 51 \mathrm{~N} 55^{\circ} \mathrm{E}$ & $51^{\circ} 82 \mathrm{~N}^{\circ} 35 \mathrm{E}$ & $59^{\circ} 49 \mathrm{~N} 17^{\circ} 38 \mathrm{E}$ & $48^{\circ} 48 \mathrm{~N} 2^{\circ} 08 \mathrm{E}$ \\
\hline First year of bare fallow & 1956 & 1959 & 1959 & 1956 & 1928 \\
\hline Last sampling for this study & 1983 & 2007 & 2008 & 2009 & 2008 \\
\hline Duration (years) & 27 & 48 & 49 & 53 & 79 \\
\hline Previous land use & arable & grassland & grassland & arable & grassland \\
\hline Mean annual temperature $\left({ }^{\circ} \mathrm{C}\right)$ & 7.8 & 10.7 & 9.5 & 5.5 & 10.7 \\
\hline Annual precipitation (mm) & 862 & 649 & 712 & 533 & 628 \\
\hline $\begin{array}{l}\text { Soil texture and type IUSS } \\
\text { Working Group WRB, } 2006\end{array}$ & $\begin{array}{l}\text { Coarse sandy } \\
\text { loam Orthic Luvisol }\end{array}$ & Silty loam Luvisol & $\begin{array}{l}\text { Flinty silty clay loam Chromic } \\
\text { Luvisol }\end{array}$ & Clay loam Eutric Cambisol & Silty loam Luvisol \\
\hline Sampling depth (cm) & 20 & 25 & 23 & 20 & 25 \\
\hline Initial SOC ( $\left.\mathrm{g} \mathrm{kg}^{-1}\right)$ & 16.8 & 13.6 & 29.5 & 14.5 & 17.9 \\
\hline Final SOC $\left(\mathrm{g} \mathrm{kg}^{-1}\right)$ & 11.0 & 8.6 & 10.0 & 8.9 & 6.0 \\
\hline
\end{tabular}


in the bulk density of the soils. The fire history at those sites is not known in detail but the PyOC measured in this study most probably comes from the burning of herbaceous, scrubs and other low woodland vegetation during the century before the fallow treatment (based on the history of the sites and the distribution of the different BPCA molecular markers). The soils at Askov and Ultuna are known to have been under arable management for centuries before the start of the bare-fallow experiments in 1956.

\section{PyOC Assessment}

Revealing the dynamics of PyOC requires a reliable method for its determination in soil. Several methods have been proposed
(Hammes et al., 2007). The Benzene Polycarboxylic Acid (BPCA) molecular marker method (Glaser et al., 1998; Wiedemeier et al., 2013) has the advantage of only targeting pyrogenic derived molecular markers, thus yielding conservative but quantitative estimates of $\mathrm{PyOC}$ as well as information about its quality (Wiedemeier et al., 2015). The BPCA method is, to date, the most widely used approach to measure PyOC contents in soils (Reisser et al., 2016).

PyOC contents were assessed with the BPCA method following the previously described state-of-the-art molecular marker approach for PyOC analysis in environmental samples (Wiedemeier et al., 2013). Samples were analyzed in duplicate and analytical error was always $<10 \%$. For

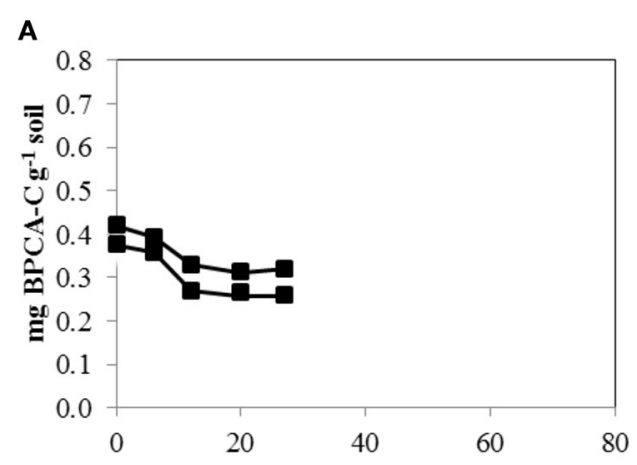

B

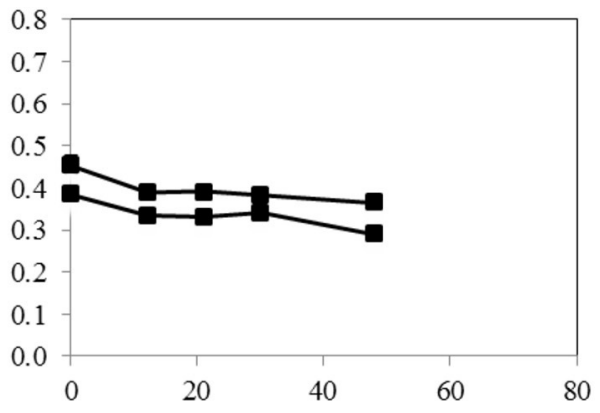

C

D
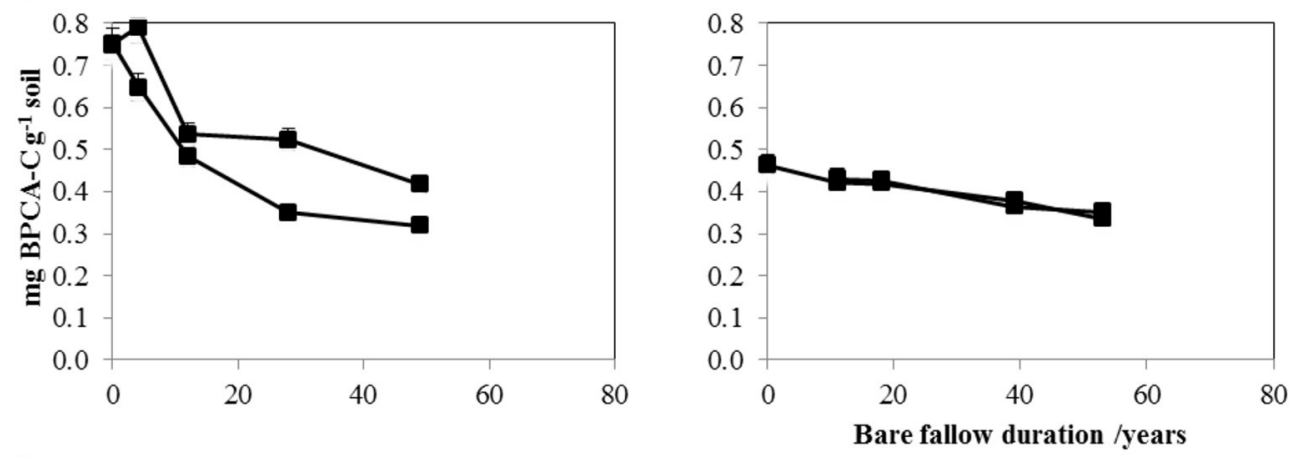

E

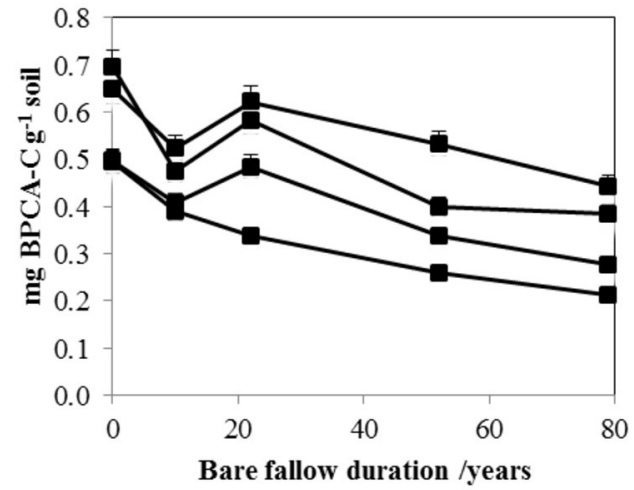

FIGURE 1 | Contents of native pyrogenic organic carbon (PyOC) in soil decrease with time at all LTBF sites and for all field replicates (mg BPCA-C $\mathrm{g}^{-1}$ soil, time in years): Askov (A), Grignon (B), Rothamsted (C), Ultuna (D), and Versailles (E). Each line corresponds to an experimental plot. Error bars represent PyOC variability within analytical replicates (always $<10 \%)$. 
each analytical batch of soils, a laboratory reference soil (Chernozem) was included to ensure the quality of the analytical procedures. The BPCA method is consistent but probably underestimates the absolute amount of PyOC (Glaser et al., 1998), and a conversion factor of 2.27 has been proposed to convert "BPCA-PyOC" to "total-PyOC." We refrained from this and present unconverted PyOC-values because only relative changes in the PyOC content were of interest.

\section{SOC Assessment}

The content of SOC in the samples was measured by dry combustion employing a CHN Autoanalyser (Carlo Erba NA 1500). Except for Grignon, the soils contained no carbonates, therefore total $\mathrm{C}$ is equivalent to organic $\mathrm{C}$ contents. The Grignon soils were treated with $\mathrm{HCl}$ to remove soil carbonates (Harris et al., 2001).

\section{Statistical Analysis}

The statistical analyses were conducted using the free software environment for statistical computing R (http://www.r-project. org).

\section{One Pool Model}

In a first step we unsuccessfully tried to fit a two pool model on the experimental data. Indeed, because of the small number of date points for each experimental plot, five dates per plot, we were unable to constrain the model parameters (see Supplementary Table 1). We therefore decided to use a simpler model to estimate and compare the MRT of both PyOC and SOC.

Although simplistic, single pool models have been used to describe SOC dynamics (Parton et al., 1988) and allow a direct and transparent comparison between PyOC and SOC dynamics and between our dataset and previously published data. This model assumes that the loss of PyOC can be described by first

TABLE 2 | PyOC contents (mg PyOC-C g ${ }^{-1}$ soil) measured by the BPCA method and coefficient of variation CV (\%) for all samples and all sites

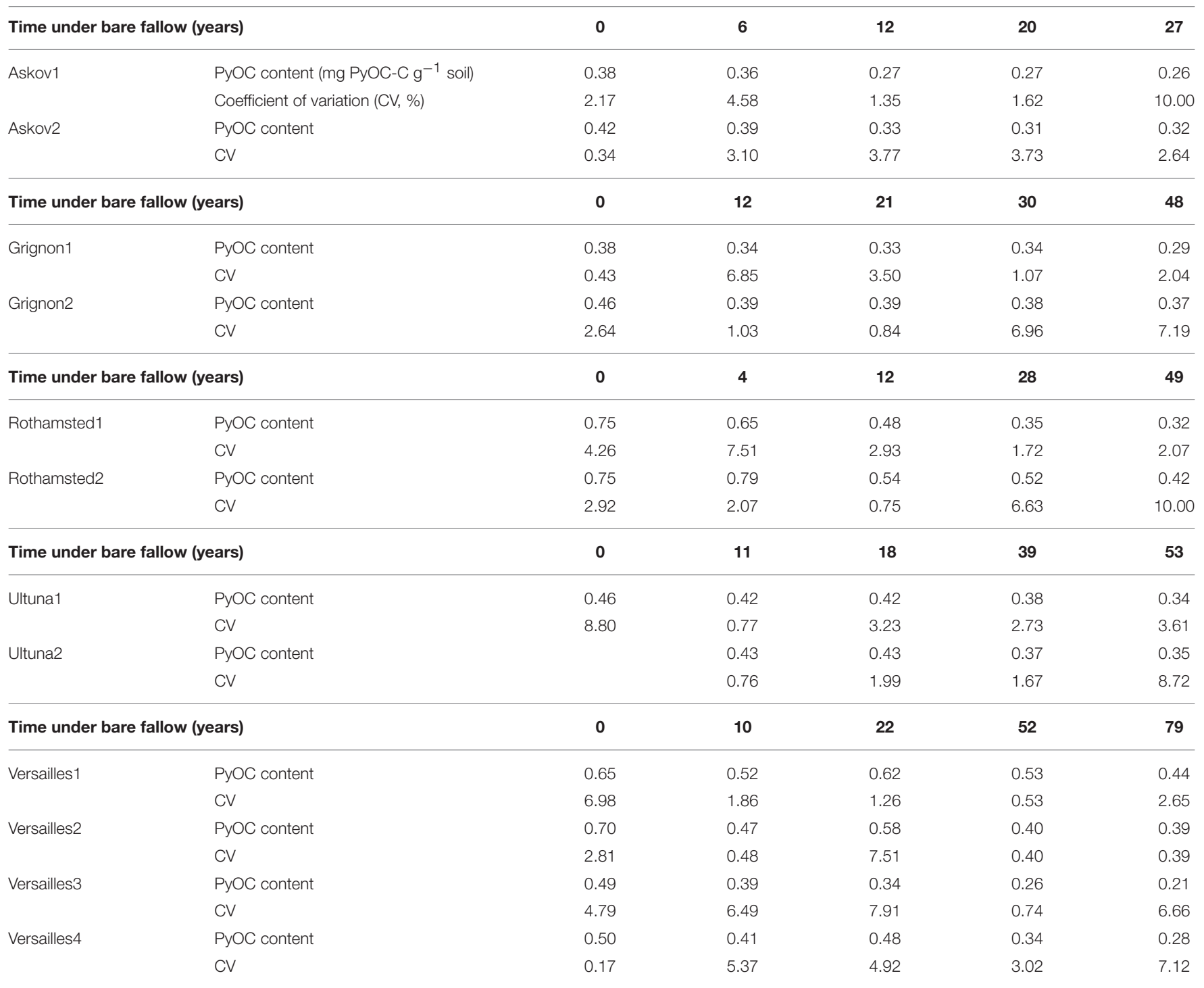


order kinetics:

$$
\mathrm{C}(\mathrm{t})=\mathrm{C}(0)^{*} \exp (-\mathrm{t} / \mathrm{MRT})
$$

Where $\mathrm{C}(\mathrm{t})$ is concentration of PyOC (mg PyOC-C $\mathrm{g}^{-1}$ soil) at time $\mathrm{t}$ (years after the bare fallow start), $\mathrm{C}(0)$ is the initial PyOC concentration (mg PyOC-C $\mathrm{g}^{-1}$ soil), and MRT is the Mean Residence Time of PyOC (years). The same model was used to assess the MRT of SOC. The Least Square method was used to estimate model parameters.

\section{RESULTS}

\section{PyOC Contents Decrease Rapidly}

We found contents of soil PyOC detected by the BPCA method to decrease surprisingly fast at all LTBF sites (Figure $\mathbf{1}$ and Table 2). Initial concentrations varied between $0.38 \mathrm{mg}$ PyOC$\mathrm{C} \mathrm{g}^{-1}$ soil (plots Grignon1 and Askov1) and $0.75 \mathrm{mg}$ PyOC$\mathrm{C}^{-1}$ soil (Rothamsted1 and 2) corresponding to 2 and $4 \%$ of the total organic carbon (TOC) contents (TOC $=$ PyOC + SOC). This is consistent with results from other studies using similar methods (Glaser et al., 1998; Schneider et al., 2011). For all LTBF sites, the final PyOC concentrations were significantly lower than the initial concentrations $(p<0.001)$. The loss of PyOC between the first and the last soil sampling ranged from 19.8 to $57.3 \%$ of the initial PyOC content but was not related to the length of the bare fallow period. At Versailles and Rothamsted, PyOC contents showed steep initial declines, with $20-35 \%$ of the initial PyOC being lost during the first decade, corresponding to average annual losses of $1.35 \%$ at Versailles and $2 \%$ at Rothamsted. The PyOC increased between year 10 and year 22 in soils from the Versailles bare fallow, probably due to the impact of nearby bombings during the Second World War
(WW2). In the longest running field experiment (Versailles), 55\% $(S D=10 \%)$ of the initial PyOC remained after 80 years of bare fallow.

\section{PyOC Becomes Enriched in Condensed Material}

The evolution of the quantities of the different BPCA molecular markers showed a consistent decrease of the ratio B5CA:B6CA with time for all sites and all replicates (Figure 2). This translates into a relative increase in the quantity of B6CAs, which are the most condensed BPCA molecular markers.

\section{PyOC and SOC Loss Differs Little}

As previously reported (Barré et al., 2010), TOC contents decreased at all LTBF sites due to the absence of $\mathrm{C}$ inputs and the on-going decomposition of organic matter formed during the pre-bare fallow period. At the last sampling, between 34 and $72 \%$ of the initial SOC was lost (Table 1 and Figure 3).

The PyOC and SOC pools showed similar dynamics (Figure 4) and the PyOC loss patterns were consistent across the range of pedo-climatic settings. Loss rates of PyOC were significantly smaller than those observed for SOC $(p<0.001)$. The difference between PyOC and SOC loss rates was reflected in a relative PyOC enrichment at all LTBF sites; the initial PyOC content ranged from 2 to $4 \%$ of the SOC while final PyOC contents ranged from 2 to $6 \%$ of the SOC. At Rothamsted, one sample taken 4 years after the beginning of the bare fallow contained more PyOC than the initial amount at the beginning of the bare fallow (Table 2). This outlier, ascribed to spatial heterogeneity, is thus located above the $100 \%$ line of PyOC in Figure 4, but remains within the $10 \%$ variation.

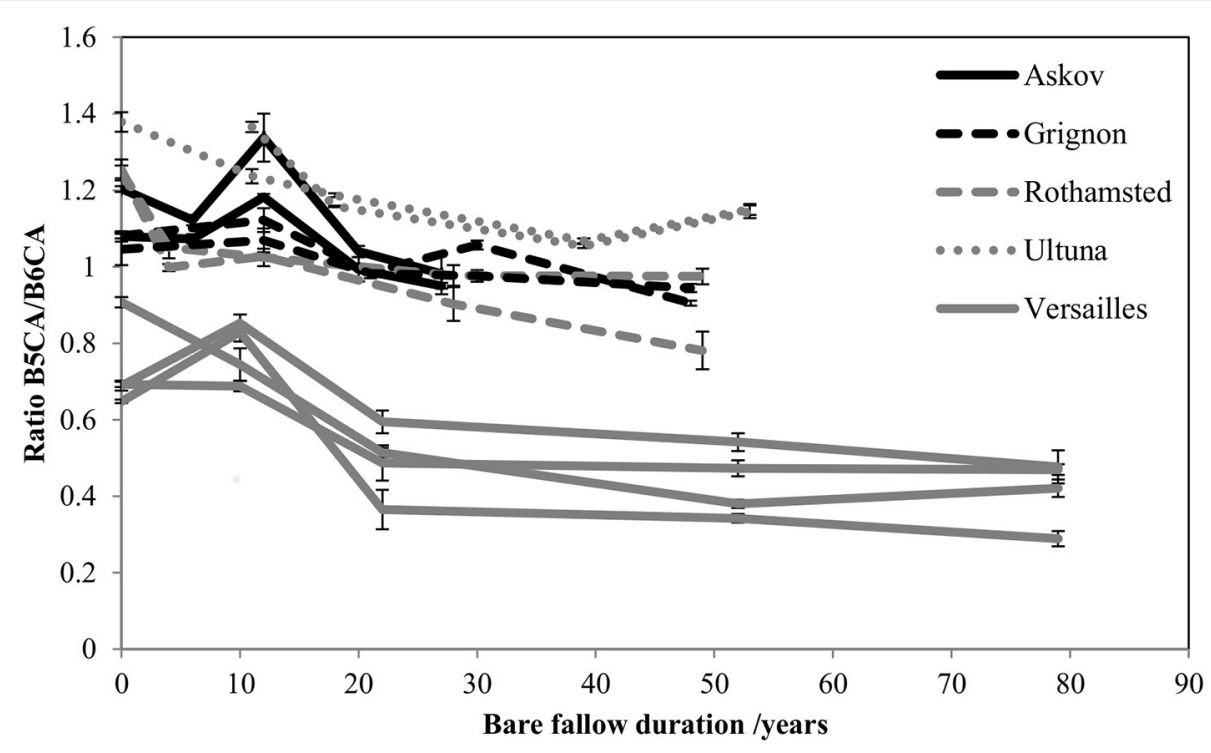

FIGURE 2 | Consistent qualitative evolution of PyOC at all LTBF sites. Evolution of the ratio between the quantities (mg PyOC-C $\mathrm{g}^{-1}$ soil) of two molecular markers (B5CA and B6CA) out of the four molecular markers analyzed with the BPCA method. Error bars represent the variability observed between technical replicates. 

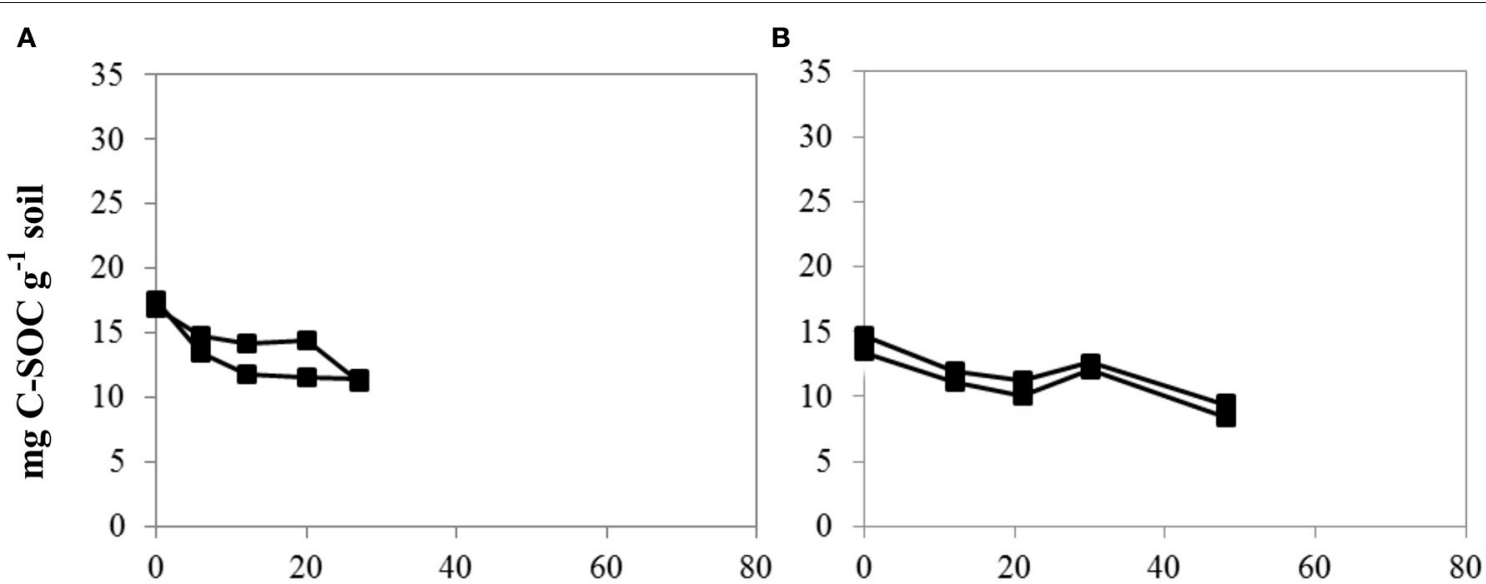

C

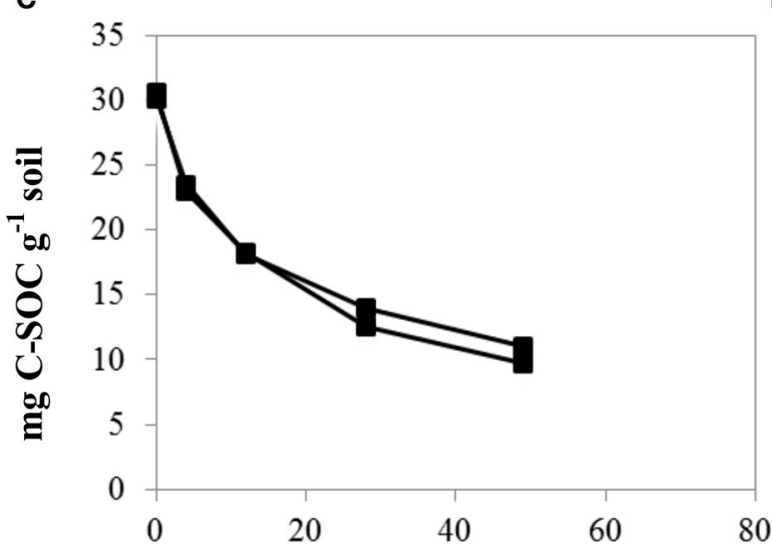

E

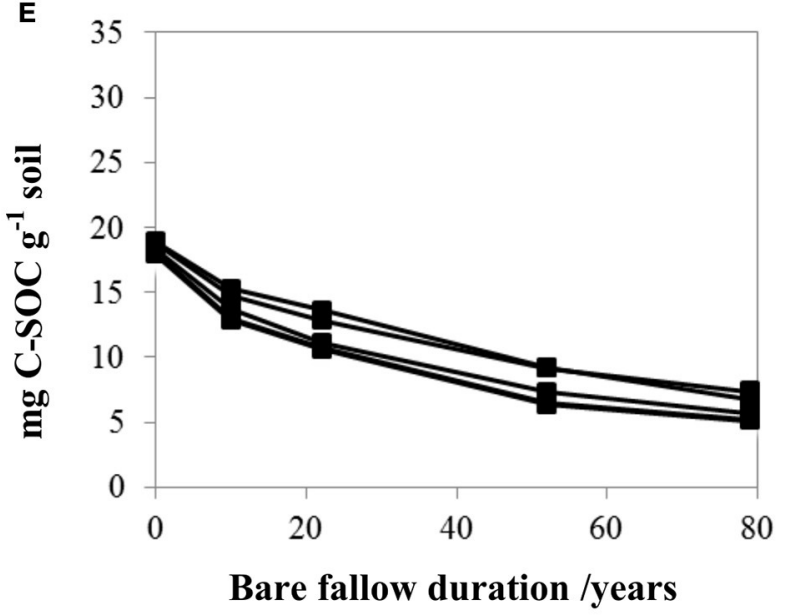

D

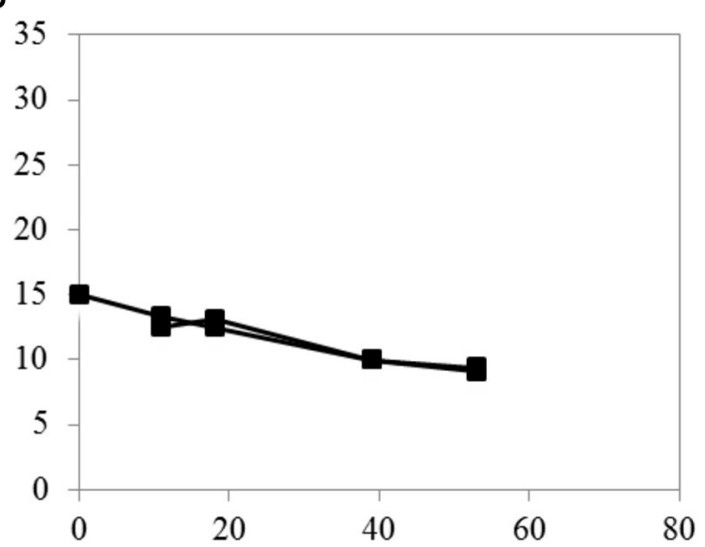

Bare fallow duration /years

FIGURE 3 | SOC degradation dynamics with time at all sites (mg C. $\mathrm{g}^{-1}$ soil) for all field replicates: Askov (A), Grignon (B), Rothamsted (C), Ultuna (D), and Versailles (E). SOC represents the portion of total organic carbon which is not pyrogenic, SOC contents are calculated as the difference between TOC contents determined by elemental analysis and PyOC contents determined by BPCA analysis.

\section{PyOC Residence Time Ranges from 42 to 183 Years}

The MRT for PyOC and SOC was estimated using first order kinetics in order to compare the relative persistence of PyOC and SOC (Table 3). After unsuccessfully trying to model our data using a two pool model, we applied single pool models with mono-exponential decay assuming PyOC and SOC to be homogeneous pools. 


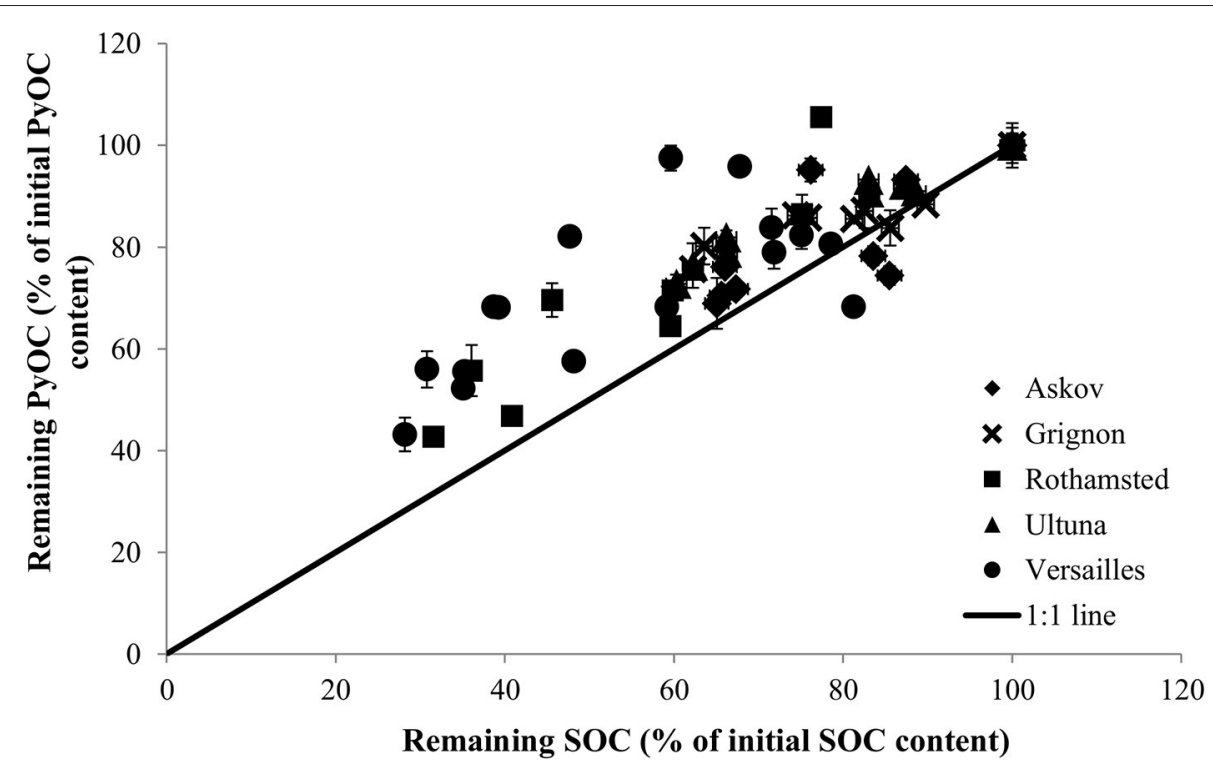

FIGURE 4 | PyOC losses occur more slowly than SOC losses (1:1 line), far from the concept of PyOC being inert (0:1 line). Values are expressed as \% of initial SOC and PyOC contents. Time series start at the 100\% values for both PyOC and SOC, vertical error bars represent PyOC variability within analytical replicates (<10\%), horizontal error bars represent analytical errors on SOC measurements ( $<3 \%)$. The average annual loss of SOC ranged from $0.7 \%$ at Ultuna to $1.4 \%$ at Rothamsted while losses of PyOC varied from $0.4 \%$ at Versailles to $1.2 \%$ at Rothamsted.

The MRT obtained (Table 3) represent the average time an atom of $\mathrm{C}$ in PyOC or in SOC remains in the PyOC or SOC reservoirs. For instance in Rothamsted, modeling results show that PyOC has a MRT of 42 years $(S D=7$ years) and 76 years $(S D$ $=15$ years) in the two plots studied (Table 3 ). This means that part of the PyOC material is expected to persist for time periods well exceeding the calculated MRT. Details of the calculations of the age distribution of material with a one pool model can be found in Manzoni et al. (2009). Given the results of the model, even though MRT of PyOC is 80 years for the Versailles3 plot, after 240 years of LTBF there would still be $5 \%$ of the initial PyOC remaining (Supplementary Figure 1).

Excluding data from the three Versailles plots distorted by bombing impacts, the MRT of PyOC ranged from 42 to 183 years, with an average of 116 years across all sites (the standard deviation is 15 years). The average MRT for PyOC (116 years) is 1.6 times longer than the MRT estimated for SOC (73 years). This difference is significant $(p<0.001)$ and falls in the low end of estimates (decades to centuries) reported by the Intergovernmental Panel on Climate Change (IPCC) (Ciais et al., 2013).

The ratio between MRT of PyOC and SOC differed among LTBF sites. For the Askov2 plot, the MRT of PyOC and SOC are the same, evidencing no specific PyOC persistence, while for the Versailles1 plot the MRT of PyOC was three times larger than that of SOC. Despite large differences in the pedo-climatic setting among the LTBF sites, no relationship could be established between PyOC loss and general site characteristics, such as soil type and climate. However, sites with a pre-fallow history of permanent grasslands (Rothamsted and Versailles) showed a steeper initial decline in PyOC than sites with a long pre-fallow history of arable land use (Askov and Ultuna).

\section{DISCUSSION}

The small difference in PyOC and SOC persistence contrasts with current concepts on this topic. The MRT of SOC in the LTBF plots are similar to those estimated from alternative approaches, such as C3-C4 vegetation conversion experiments under similar pedo-climatic conditions (Dignac et al., 2005; Rasse et al., 2006) and also to those estimated from similar approaches using more complex models (Barré et al., 2010; Cardinael et al., 2015). Barré et al. (2010) have extensively studied the dynamics of TOC in six long term bare fallows including the five sites of the present study. They present numerical results of turnover times, equivalent to MRT in this study, using a two pool model (mono exponential + constant). They found turnover times ranging from 24 years at Versailles to 66 years at Ultuna with an average of 47 years, for what they refer to as the intermediate pool of their two pool model. In our study, the MRT of SOC are within the same order of magnitude but tend to be bigger, ranging from 31 years in Rothamsted to 111 years in Grignon and averaging at 73 years. Therefore, by using a one pool model we overestimated the MRT of SOC. In the same way, if similar models had been fitted to PyOC dynamics, they probably would have led to smaller MRT for PyOC compared to the results presented in this study.

The MRT for PyOC in the soils from these five North European sites are three to ten times shorter than MRT values for PyOC often reported in the literature for long-term field studies (Singh et al., 2012a; Lehmann et al., 2015), except for Bird et al. (1999) and Nguyen et al. (2009) which found comparable MRT of 130 and 141 years, respectively for a chronosequence in tropical contexts. Litter inputs, which do not occur in LTBF soils, might have had an impact, either positive or negative on PyOC dynamics: priming effect could have resulted in a quicker decline 
TABLE 3 | Mean residence time (in years) for PyOC and SOC calculated from the whole data set and estimates from previous studies.

\begin{tabular}{|c|c|c|c|c|c|}
\hline & \multicolumn{2}{|c|}{$\begin{array}{c}\text { MRT PyOC } \\
\text { (years) }\end{array}$} & \multicolumn{2}{|c|}{$\begin{array}{c}\text { MRT SOC } \\
\text { (years) }\end{array}$} & \multirow[t]{2}{*}{$\begin{array}{l}\text { Ratio MRT } \\
\text { PyOC/SOC }\end{array}$} \\
\hline & mean & $S D$ & mean & $S D$ & \\
\hline Askov1 & 59 & 9 & 46 & 8 & 1.3 \\
\hline Askov2 & 76 & 10 & 77 & 11 & 1.0 \\
\hline Grignon1 & 183 & 22 & 111 & 25 & 1.6 \\
\hline Grignon2 & 169 & 31 & 109 & 19 & 1.5 \\
\hline Rothamsted1 & 42 & 7 & 31 & 5 & 1.3 \\
\hline Rothamsted2 & 76 & 15 & 35 & 6 & 2.2 \\
\hline Ultuna1 & 183 & 11 & 99 & 3 & 1.8 \\
\hline Ultuna2 & 179 & 18 & 95 & 13 & 1.9 \\
\hline Versailles1 & $214^{*}$ & 53 & $70^{\star}$ & 8 & $3.1^{\star}$ \\
\hline Versailles2 & $106^{\star}$ & 27 & $71^{*}$ & 4 & $1.5^{\star}$ \\
\hline Versailles3 & 80 & 9 & 49 & 6 & 1.6 \\
\hline Versailles4 & $141^{\star}$ & 25 & $54^{\star}$ & 6 & $2.6^{\star}$ \\
\hline Average & $116\left(126^{\star \star}\right)$ & $15\left(20^{\star \star}\right)$ & $73\left(72^{\star \star}\right)$ & $10\left(10^{\star \star}\right)$ & $1.58\left(1.8^{\star \star}\right)$ \\
\hline Singh et al., 2012a & $291\left(353^{\star \star \star}\right)$ & & - & & \\
\hline $\begin{array}{l}\text { Lehmann et al., } \\
2015\end{array}$ & 300-6,000 & & - & & \\
\hline Forbes et al., 2006 & $>1,000$ & & - & & \\
\hline
\end{tabular}

The significance of the model is shown in the standard deviation (SD) of each MRT estimates (in years).

*Data impacted by WW2 bombing event at Versailles.

**Including hampered data.

${ }^{\star \star \star}$ For field studies.

of PyOC (Kuzyakov, 2010) whereas preferential biodegradation of fresh litter could have slowed down the degradation of PyOC. However, published evidence suggests that priming effect is not a significant factor for explaining long term soil organic carbon dynamics (Cardinael et al., 2015). Additionnally, Vasilyeva et al. (2011) compared the PyOC contents of bare fallow soils (after 55 years of bare fallow) to the PyOC contents of soils from an adjacent native grass steppe. They found that the absence of vegetation did not interact with the persistence of PyOC in soil as the observed changes in PyOC contents were similar for fallowed and vegetated soils. For all these reasons, our results, i.e., relatively short MRT for PyOC, are likely not due to the fact that the considered soils were kept bare.

Losses of PyOC are ascribed to biotic (microbial degradation) and abiotic mechanisms (photo-oxidation, erosion, and leaching). We consider that the consistent loss of PyOC observed across the contrasted pedo-climatic settings is mainly due to biotic processes. Except for Rothamsted, no significant soil bulk density changes were observed in the different LTBF sites, the same soil layers were therefore consistently sampled over the duration of the experiments (see Materials and Methods). Photo-oxidation impacts charred materials situated at the soil surface, but can only be of minor importance in our study since soil samples were extracted from the Ap-horizon (0-20 or $0-30 \mathrm{~cm}$ soil depth depending on the site). Furthermore, the experimental setup of the LTBF experiments ensured that losses of PyOC and SOC by horizontal transport (e.g., erosion) were insignificant during the fallow period. Although migration of PyOC may occur, previous studies of potential loss mechanisms found that migration of soluble PyOC in the soil profile plays an insignificant role compared to biological degradation (Major et al., 2010; Abiven et al., 2011; Maestrini et al., 2014). Moreover, similar changes in PyOC molecular characteristics were observed across sites (Figure 2), supporting the idea that biological decay was the dominating mechanism for the observed loss of PyOC.

In their recommendations for future estimations of $\mathrm{PyOC}$ MRT, Lehmann et al. (2015) recommended considering at least a two pool model, to take into account the heterogeneity of the PyOC material. Despite our large dataset, it was not possible to fit any multiple pool models to the five data chronicles, which might lead one to question the accuracy of this recommendation. Since our dataset is particularly large, in particular along time thanks to the archived samples, it is very unlikely to find better time-framed setups to estimate MRT. Instead of increasing the complexity of the model with poorly constrained parameters, it would be more useful to always compare the PyOC MRT to the SOC MRT of the same site as a way to normalize the persistence of the organic materials. Even though our results might represent minimum estimates of MRT since one pool models do not consider long term pools, our study is to our knowledge the first direct comparison between PyOC and SOC persistence in temperate arable soils. It conveys new and essential experimental evidence on the stability of native PyOC and expands current concepts of PyOC persistence in soil. We found PyOC to be more persistent than SOC and as such, our results align with the current notion of PyOC being the most persistent fraction of TOC (Schmidt et al., 2002; Amelung et al., 2008). While our results may be consistent with this notion, the relatively short MRT observed for PyOC in LTBF soils suggest that PyOC persistence might have been overestimated in previous reports.

\section{AUTHOR CONTRIBUTIONS}

SL, CC, PB, and SA: designed the study and wrote the paper; SL: carried out analyses; DW developed the improved BPCA method and provided technical advice; FvO, AM, TK, SH, and BC: provided the LTBF soil samples. All authors subsequently reviewed and amended the manuscript.

\section{ACKNOWLEDGMENTS}

We gratefully acknowledge the colleagues and the institutions who initiated and maintained the LTBF experiments and who thereby made it possible for us to undertake this study. We thank ABIES doctoral school and the European Climate KIC mobility program for funding the missions of SL to the University of Zurich. We thank Rothamsted Research and the Lawes Agricultural Trust for access to archived soil samples from their long-term experiments. The Rothamsted Longterm Experiments National Capability is supported by the UK Biotechnology and Biological Sciences Research Council and 
the Lawes Agricultural Trust. The Danish contribution was financially supported by the EU FP7 project SmartSOIL (Grant agreement 289694). The Swiss contribution was supported by the Zurich University Research Priority Program "Global Change and Biodiversity."

\section{REFERENCES}

Abiven, S., Hengartner, P., Schneider, M. P. W., Singh, N., and Schmidt, M. W. I. (2011). Pyrogenic carbon soluble fraction is larger and more aromatic in aged charcoal than in fresh charcoal. Soil Biol. Biochem. 43, 1615-1617. doi: 10.1016/j.soilbio.2011.03.027

Alexis, M. A., Rasse, D. P., Knicker, H., Anquetil, C., and Rumpel, C. (2012). Evolution of soil organic matter after prescribed fire: a 20-year chronosequence. Geoderma 189, 98-107. doi: 10.1016/j.geoderma.2012.05.003

Amelung, W., Brodowski, S., Sandhage-Hofmann, A., and Bol, R. (2008). Chapter 6: Combining biomarker with stable isotope analyses for assessing the transformation and turnover of soil organic matter. Adv. Agron. 100, 155-250. doi: 10.1016/S0065-2113(08)00606-8

Barré, P., Eglin, T., Christensen, B. T., Ciais, P., Houot, S., Kätterer, T., et al. (2010). Quantifying and isolating stable soil organic carbon using long-term bare fallow experiments. Biogeosciences 7, 3839-3850. doi: 10.5194/bg-7-3839-2010

Bird, M. I., Moyo, C., Veenendaal, E. M., Lloyd, J., and Frost, P. (1999). Stability of elemental carbon in a savanna soil. Glob. Biogeochem. Cycles 13, 923-932. doi: 10.1029/1999GB900067

Cardinael, R., Eglin, T., Guenet, B., Neill, C., Houot, S., and Chenu, C. (2015). Is priming effect a significant process for long-term SOC dynamics? Analysis of a 52-years old experiment. Biogeochemistry 123, 203-219. doi: 10.1007/s10533-014-0063-2

Ciais, P., Sabine, C., Bala, G., Bopp, L., Brovkin, V., Canadell, J., et al. (2013). "Carbon and other biogeochemical cycles," in Climate Change 2013: The Physical Science Basis, Contribution of Working Group I to the Fifth Assessment Report of the Intergovernmental Panel on Climate Change, eds T. F. Stocker, D. Qin, G. K. Plattner, M. Tignor, S. K. Allen, J. Boschung, A. Nauels, Y. Xia, V. Bex, and P. M. Midgley (Cambridge; New York, NY: Cambridge University Press).

Dignac, M.-F., Bahri, H., Rumpel, C., Rasse, D. P., Bardoux, G., Balesdent, J., et al. (2005). Carbon-13 natural abundance as a tool to study the dynamics of lignin monomers in soil: an appraisal at the Closeaux experimental field (France). Geoderma 128, 3-17. doi: 10.1016/j.geoderma.2004.12.022

Forbes, M. S., Raison, R. J., and Skjemstad, J. O. (2006). Formation, transformation and transport of black carbon (charcoal) in terrestrial and aquatic ecosystems. Sci. Total Environ. 370, 190-206. doi: 10.1016/j.scitotenv.2006.06.007

Glaser, B., Haumaier, L., Guggenberger, G., and Zech, W. (1998). Black carbon in soils: the use of benzenecarboxylic acids as specific markers. Org. Geochem. 29, 811-819. doi: 10.1016/S0146-6380(98)00194-6

Hammes, K., Schmidt, M. W. I., Smernik, R. J., Currie, L. A., Ball, W. P., Nguyen, T. H., et al. (2007). Comparison of quantification methods to measure fire-derived (black/elemental) carbon in soils and sediments using reference materials from soil, water, sediment and the atmosphere. Glob. Biogeochem. Cycles 21:GB3016. doi: 10.1029/2006GB002914

Harris, D., Horwáth, W. R., and van Kessel, C. (2001). Acid fumigation of soils to remove carbonates prior to total organic carbon or CARBON-13 isotopic analysis. Soil Sci. Soc. Am. J. 65:1853. doi: 10.2136/sssaj.2001.1853

IUSS Working Group WRB (2006). World Reference Base for Soil Resources. Rome: FAO.

Kuzyakov, Y. (2010). Priming effects: interactions between living and dead organic matter. Soil Biol. Biochem. 42, 1363-1371 doi: 10.1016/j.soilbio.2010.04.003

Lehmann, J., Abiven, S., Kleber, M., Genxing, P., Singh, B., Sohi, S., et al. (2015). "Persistence of biochar in soil," in Biochar for Environmental Management, eds J. Lehmann and S. Joseph (London; New York, NY: Routledge).

Lehmann, J., Skjemstad, J., Sohi, S., Carter, J., Barson, M., Falloon, P., et al. (2008). Australian climate-carbon cycle feedback reduced by soil black carbon. Nat. Geosci. 1, 832-835. doi: 10.1038/ngeo358

\section{SUPPLEMENTARY MATERIAL}

The Supplementary Material for this article can be found online at: https://www.frontiersin.org/articles/10.3389/feart. 2017.00096/full\#supplementary-material

Lehndorff, E., Roth, P. J., Cao, Z. H., and Amelung, W. (2014). Black carbon accrual during 2000 years of paddy-rice and non-paddy cropping in the Yangtze River Delta, China. Glob. Change Biol. 20, 1968-1978. doi: 10.1111/gcb.12468

Maestrini, B., Abiven, S., Singh, N., Bird, J., Torn, M. S., and Schmidt, M. W. I. (2014). Carbon losses from pyrolysed and original wood in a forest soil under natural and increased N deposition. Biogeosciences 11, 5199-5213. doi: 10.5194/bg-11-5199-2014

Major, J., Lehmann, J., Rondon, M., and Goodale, C. (2010). Fate of soilapplied black carbon: downward migration, leaching and soil respiration. Glob. Change Biol. 16, 1366-1379. doi: 10.1111/j.1365-2486.2009. 02044.x

Manzoni, S., Katul, G. G., and Porporato, A. (2009). Analysis of soil carbon transit times and age distributions using network theories, J. Geophys. Res. 114:G04025. doi: 10.1029/2009JG001070

Nguyen, B. T., Lehmann, J., Kinyangi, J., Smernik, R., Riha, S. J., and Engelhard, M. H. (2009). Long-term black carbon dynamics in cultivated soil. Biogeochemistry 92, 163-176. doi: 10.1007/s10533-008-9248-x

Paradelo, R., van Oort, F., and Chenu, C. (2013). Water-dispersible clay in bare fallow soils after 80 years of continuous fertilizer addition. Geoderma 200-201, 40-44. doi: 10.1016/j.geoderma.2013. 01.014

Parton, W. J., Stewart, J. W. B., and Cole, C. V. (1988). 357 Dynamics of C, N, P and S in grassland soils: a model. Biogeochemistry 5, 109-131. doi: 10.1007/BF02180320

Rasse, D. P., Mulder, J., Moni, C., and Chenu, C. (2006). Carbon turnover kinetics with depth in a french loamy soil. Soil Sci. Soc. Am. J. 70:2097. doi: 10.2136/sssaj2006.0056

Reisser, M., Purves, R. S., Schmidt, M. W. I., and Abiven, S. (2016). Pyrogenic carbon in soils: a literature-based inventory and a global estimation of its content in soil organic carbon and stocks. Front. Earth Sci. 4:80. doi: 10.3389/feart.2016.00080

Rühlmann, J. (1999). A new approach to estimating the pool of stable organic matter in soil using data from long-term field experiments. Plant Soil 213, 149-160. doi: 10.1023/A:1004552016182

Schmidt, M. W. I., Skjemstad, J. O., and Jäger, C. (2002). Carbon isotope geochemistry and nanomorphology of soil black carbon: black chernozemic soils in central Europe originate from ancient biomass burning. Glob. Biogeochem. Cycles 16:1123. doi: 10.1029/2002GB001939

Schneider, M. P. W., Smittenberg, R. H., Dittmar, T., and Schmidt, M. W. I. (2011). Comparison of gas with liquid chromatography for the determination of benzenepolycarboxylic acids as molecular tracers of black carbon. Org. Geochem. 42, 275-282. doi: 10.1016/j.orggeochem.2011.01.003

Sierra, C. A., Müller, M., Metzler, H., Manzoni, S., and Trumbore, S. E. (2017). The muddle of ages, turnover, transit, and residence times in the carbon cycle. Glob. Change Biol. 23, 1763-1773. doi: 10.1111/gcb.13556

Singh, N., Abiven, S., Torn, M. S., and Schmidt, M. W. I. (2012a). Fire-derived organic carbon in soil turns over on a centennial scale. Biogeosciences 9, 2847-2857. doi: 10.5194/bg-9-2847-2012

Singh, B. P., Cowie, A. L., and Smernik, R. J. (2012b). Biochar carbon stability in a clayey soil as a function of feedstock and pyrolysis temperature. Environ. Sci. Technol. 46, 11770-11778. doi: 10.1021/es302545b

Vasilyeva, N. A., Abiven, S., Milanovskiy, E. Y., Hilf, M., Rizhkov, O. V., and Schmidt, M. W. I. (2011). Pyrogenic carbon quantity and quality unchanged after 55 years of organic matter depletion in a Chernozem. Soil Biol. Biochem. 43, 1985-1988. doi: 10.1016/j.soilbio.2011.05.015

Whitman, T., Enders, A., and Lehmann, J. (2014). Pyrogenic carbon additions to soil counteract positive priming of soil carbon mineralization by plants. Soil Biol. Biochem. 73, 33-41. doi: 10.1016/j.soilbio.2014.02.009 
Wiedemeier, D. B., Abiven, S., Hockaday, W. C., Keiluweit, M., Kleber, M., Masiello, C. A., et al. (2015). Aromaticity and degree of aromatic condensation of char. Org. Geochem. 78, 135-143. doi: 10.1016/j.orggeochem.2014.10.002

Wiedemeier, D. B., Hilf, M. D., Smittenberg, R. H., Haberle, S. G., and Schmidt, M. W. I. (2013). Improved assessment of pyrogenic carbon quantity and quality in environmental samples by high-performance liquid chromatography. J. Chromatogr. A 1304, 246-250. doi: 10.1016/j.chroma.2013. 06.012

Zimmerman, A. R. (2010). Abiotic and microbial oxidation of laboratoryproduced black carbon (Biochar). Environ. Sci. Technol. 44, 1295-1301. doi: $10.1021 / \mathrm{es} 903140 \mathrm{c}$
Conflict of Interest Statement: The authors declare that the research was conducted in the absence of any commercial or financial relationships that could be construed as a potential conflict of interest.

Copyright $\odot 2017$ Lutfalla, Abiven, Barré, Wiedemeier, Christensen, Houot, Kätterer, Macdonald, van Oort and Chenu. This is an open-access article distributed under the terms of the Creative Commons Attribution License (CC BY). The use, distribution or reproduction in other forums is permitted, provided the original author(s) or licensor are credited and that the original publication in this journal is cited, in accordance with accepted academic practice. No use, distribution or reproduction is permitted which does not comply with these terms. 